\title{
DESIGNING A CURRICULUM ABOUT ELECTRON MICROSCOPE BASED ON DISCIPLINARY METHOD FOR GRADUATE STUDENTS
}

\author{
A. Karami* and D. Badiee \\ Chemistry Department, Shahid Rajaee Teacher Training University, Tehran, Iran
}

Published online: 15 May 2016

\begin{abstract}
Nanotechnology has developed in all fields of human life and many research institutes and universities teach this major in their departments. The aim of this research was to design the educational objects and contents about identifying and characterization of nanomaterials by electron microscope for undergraduate students. Research is applied and descriptive. In this study the tool was an objective-content researcher made table. Ten books from reference books and electronic sources were selected as samples for design the syllabus. The objectives and content was developed according to disciplinary method. Finally, researchers propose 4 objectives and 10 contents about electron microscope learning for undergraduates. The main objects are: An introduction to nanomaterials, a review of optics and lenses, an overview to the optical microscope, an introduction to Electron microscope.
\end{abstract}

Keywords: Nanotechnology; electron microscope; optic; syllabus; undergraduate.

Author Correspondence, e-mail: ar_karami@srttu.edu

doi: http://dx.doi.org/10.4314/jfas.v8i3s.231

\section{INTRODUCTION}

Many modern technologies have advanced to the point that the relevant feature size is on the order of a few to a few hundreds of nanometers. A classic example is computer chips with key features that now reach the length Scale of smaller than $50 \mathrm{~nm}$. The demand for smaller feature sizes is increasing at a faster and faster pace. At the fundamental level, there is an 
urgent need to better understand the properties of materials on the nanoscale level. At the technological front, there is a strong demand to develop new techniques to fabricate and measure the properties of nanomaterials and related devices. Fortunately, significant advancement has been made over the last decade in both fronts. It has been demonstrated that materials at Nano scale have unique physical and chemical properties compared to their bulk counterparts and these properties are highly promising for a variety of technological applications. (Zhang, 2009) In nanotechnology, analysis can be made to the level of manipulating atoms, molecules and chemical bonds between them. (Funmilayo \& Abiodun-Solanke, 2014)

Nanotechnology influences almost every facet of everyday life from security to medicine. The concept of nanotechnology is that when one goes down to the bottom of things, one can discover unlimited possibilities and potential of the basic particle. Nanotechnology has wide industrial and clinical applications in: Medicine, Chemistry and environment, Energy, Information and communication, Heavy industry. (Kanaparthy \& Kanaparthy, 2011)

The smallest measurable size in analysis depends on several factors: light or electron wavelength as the measurement tool; resolution power of the human eyes; diffraction of light or electron as instrument. Visible light, which is the one our eyes are sensitive to, ranges between 400 and 700 nanometers (one nanometer is one billionth of a meter). By observation of materials using visible waves, only the particles greater than $400 \mathrm{~nm}$ can be observed, but considering the diffraction of light on the human eyes, and also the size of the retinal cells, the smallest size objects visually detectable to human naked eye are 75 micrometers. (Waldman, 2002). In the first attempt, scientists constructed optical microscopes which common microscope magnify the objects 1000 times .This is possible to confuse us that we can observe $75 \mathrm{~nm}$ particles, but we cannot never observe the particles with dimensions smaller than the visible light wavelength. Therefore, the minimum particle size is 0.4 micrometers (or $400 \mathrm{~nm}$ ) with the best optical microscope in theory. However, the size of the smallest features that we can distinguish under the microscope is on the order of the wavelength of the light used. This means that we cannot observe things that are smaller than a few hundred nanometers using our eyes and visible light. Because of this constraint, we have to use electron microscope (EM).

In 1930, shortly after the discovery of the particle-wave duality of electrons, (Wheeler \& Zurek, 2014). People realized that the wave characteristics of electrons could be used to build a microscope that could surpass the resolution limit of optical microscopes. In just a few 
years, the first generation of transmission electron microscopes became commercially available. However, it was not until after World War II that TEMs began to be widely used for material characterization by metallurgists and material scientists. High-resolution TEM is a technique developed since the 1970 s to image the atomic structure of materials. (Tanaka, Usukura, Kusunoki, Saito, Sasaki, Tanji, \& Arai, 2013). There are some main components to an electron microscope: an electron optical column, a vacuum system, lens supplies for focusing and deflecting the beam and the high voltage, and control software.

Electron microscopes work by using an electron beam instead of visible light and an electron detector instead of our eyes. An electron beam allows us to see at very small scales because electrons can also behave as light. It has the properties of a wave with a wavelength that is much smaller than visible light (a few trillionths of a meter!). With this wavelength we can distinguish features down to a fraction of a nanometer. (Williams \& Carter, 1996).

An electron microscope uses an 'electron beam' to produce the image of the object and magnification is obtained by 'electromagnetic fields'; unlike light or optical microscopes, in which 'light waves' are used to produce the image and magnification is obtained by a system of 'optical lenses'.

It has already been discussed that, the smaller is the wavelength of light, the greater is its resolving power. The wavelength of green light is $0.55 \mu \mathrm{m}$, in other word, it is 110,000 times longer than that of electron beam $0.05 \AA$. That is why, despite its smaller numerical aperture, an electron microscope can resolve objects as small as $0.001 \mu$ (or $10 \AA$ ), as compared to 0.2 $\mu \mathrm{m}$ by a light microscope. Thus, the resolving power of an electron microscope is 200 times greater than that of a light microscope. It produces useful magnification up to $X 400,000$, as compared to X 2000 in a light microscope. Thus, the useful magnification is 200 times greater in an electron microscope than that in a light microscope. (Egerton, 2006)

Let's explore the different types of electron microscopes, how they work and some of their applications. In a scanning electron microscope or SEM, a beam of electrons scans the surface of a sample. The electrons interact with the material in a way that triggers the emission of secondary electrons. These secondary electrons are captured by a detector, which forms an image of the surface of the sample. The direction of the emission of the secondary electrons depends on the orientation of the features of the surface. There, the image formed will reflect the characteristic feature of the region of the surface that was exposed to the electron beam. (Seiler, 1983). 
In a transmission electron microscope or TEM, a beam of electrons hits a very thin sample (usually no more than $100 \mathrm{~nm}$ thick). The electrons are transmitted through the sample. After the sample, the electrons hit a fluorescence screen that forms an image with the electrons that were transmitted. You can better understand this process by imagining how a movie projector works. In a projector, you have a film that has the negative image that will be projected. The projector shines white light on the negative and the light transmitted forms the image contained in the negative. (Harris, 2015).

TEM is widely used to observe microstructures through imaging, revealing phase/crystallographic orientation information through a diffraction pattern and discovering chemical composition by means of the energy spectrum. Like an optical microscope, a TEM can also record images of microstructures but to a much higher resolution. Typical TEM resolution can go down to the nanometer region, making it an excellent tool for nanomaterials characterization. High resolution TEM (HRTEM) can even image lattice points that are in the range of angstroms. In fact, the current resolution of TEMs is not limited by electron wavelength but by astigmatism and aberration. A huge effort is being conducted by microscopists to resolve these issues and thus push TEM's resolution further. The transmission electron aberration-corrected microscope (TEAM) project was initiated as a collaborative effort between different national labs to redesign the electron microscope around this front. (Guo \& Tan, 2009)

Since identification of nanomaterials is extremely important, students should know the principles of working with electron microscopy.

\section{METHOD}

The aim of this research was to design the educational objects and contents about identifying and characterization of nanomaterials by electron microscope for undergraduate students. Research is applied and descriptive. In this study the tool was an objective-content researcher made table. Ten books from reference books and electronic sources were selected as samples for design the syllabus.

\section{RESULT}

In this study, we proposed four topics for teaching and learning of electron microscope. First topic is introduction to the basic concepts of the nanotechnology such as: nanomaterial 
definition, some of unique features of nanomaterial, surface effect in Nano scale, different categories of nanomaterials.

In part two, students learn optic, electromagnetic waves, the "de Broglie" law, kinds of lenses, image formation, chromatic and spherical aberration in lenses.

In Part Three, Students becomes familiar with the history and invention of optical microscope and finally, should be able to explain different parts of optical microscopes, the concepts of resolution, magnification, and the limitations of optical microscopes in observation of very fine particles.

In last part, student becomes familiar with history electron microscope and how does the electron microscope work. They must exactly interpreted different parts of the electron microscope and how its work. Finally the important questions to answer: what are the advantages and disadvantages of using electron microscope?

\section{An introduction to nanomaterial}

\section{The concept of nano}

Explain the word nano.

Explain the nanoscale.

Which materials are called nanomaterials?

Define the term nanotechnology.

Name some of the applications of nanotechnology.

Name the categories of nanomaterials and give examples for each category.

\section{The importance of material properties in nanoscale}

Explain the surface effect with an example.

Why does the reactivity of the material increase in nanoscale?

How are "melting point" and speed of reactivity in nanoscale?

\section{Specifying synthesized nanomaterial}

Name some of the ways of specifying synthesized nanomaterial.

\section{An introduction to optics and lenses}

\section{Electromagnetic waves and visible region}

Define electromagnetic waves.

Specify the visible region in the electromagnetic spectrum.

Explain the relationship between wave length and energy.

Explain the "de Broglie" law. 
Describe the bilateral behavior of waves with regard to the "de Broglie" law.

\section{Concave and convex lenses and image formation}

Explain concave and convex lenses with drawing a picture.

Image formation in concave and convex lenses with drawing a picture.

Which lens is used in a microscope?

In which lens does spherical aberration happen? Why?

In which lens does chromatic aberration happen? Why?

\section{An introduction on the optical microscope}

\section{The invention of the optical microscope}

In which year was the optical microscope invented?

Who was the inventor of the optical microscope?

What structures can be seen by the optical microscope?

\section{The parts of the optical microscope}

Name different parts of the optical microscope.

Name the optical parts of the optical microscope.

name the mechanical parts of the optical microscope

Explain The status and role of the objective lens and the eyepiece

\section{Magnification and resolution}

$>$ Explain magnification and mention the formula.

Explain resolution and mention the formula.

How much is optical microscope's resolution and magnification?

Describe the limitations of the optical microscope.

\section{An introduction to Electron Microscope}

\section{The invention of the Electron microscope and its function}

In which year was the Electron microscope invented?

How does the Electron microscope work?

Explain the production of secondary electrons, backscattering electrons and X-ray.

\section{The parts of the electron microscope}

Name the 4 kinds of electron microscopes.

Name the parts of the electron microscope.

On what basis does the transmission electron microscope work? 
Name and explain different kinds of electron gun in the EM.

Describe different kinds of electromagnetic lenses.

What is the main reason for using lenses in the optic column of EM?

Demonstrate the difference between the magnetic lenses and electrostatic lenses in a table

Name the detectors and explain their relationship with different kinds of electron

Explain why it is needed to create vacuum in electron systems

What are the characteristics needed in the sample for the electron microscope in terms of thickness and conductivity?

What kind of image can we see using different kinds of electron microscopes?

What are the advantages and disadvantages of using transmission electron microscope?

\section{CONCLUSION}

After studying and analyzing valid and available sources, it was concluded that undergraduate students must primarily learn some topics to understand the characterization of nanomaterials with electron microscope. They must be aware about the entity of nanomaterial and essentiality of the knowledge of the nanomaterial. Secondly, they must be familiar with the entity of electromagnetic waves, optics, different types of concave and convex lenses and their application in optical microscope. They can convey the limitation of optical microscope in nanomaterial identification and find out the necessity of using electron microscope and understanding its different parts. They must know two types of electron microscope in nanomaterial identification. All these concepts are gathered in 4 main chapters, 10 contents and 46 behavioral education objective.

\section{REFERENCES}

[1] Aldman, G. (2002). Introduction to light: The physics of light, vision, and color. Courier Corporation.

[2] Egerton, R. (2006). Physical principles of electron microscopy: an introduction to TEM, SEM, and AEM. Springer Science \& Business Media.

[3] Funmilayo, D. I., \& Abiodun-Solanke. (2014). Nanotechnology and its Application in Dentistry. Annals of Medical and Health Sciences Research, 171-177. 
[4] Funmilayo, I. M., \& Abiodun-Solanke. (2014). Nanotechnology and its Application in Dentistry. Annals of Medical and Health Sciences Research, 171-177.

[5] Guo, Z., \& Tan, L. (2009). Fundamentals and Applications.

[6] H., C. A. (n.d.).

[7] Harris, J. R. (2015). Transmission electron microscopy in molecular structural biology: A historical survey. Archives of biochemistry and biophysics, 581, 3-18.

[8] Kanaparthy, R., \& Kanaparthy, A. (2011). The changing face of dentistry: nanotechnology. International Journal of Nanomedicine, 2799-2804.

[9] Seiler, H. (1983). Secondary electron emission in the scanning electron microscope. Journal of Applied Physics, 54(11), R1-R18.

[10]Tanaka, N., Usukura, J., Kusunoki, M., Saito, Y., Sasaki, K., Tanji, T., ... \& Arai, S. (2013). Development of an environmental high-voltage electron microscope for reaction science. Microscopy, dfs095.

[11]W Wheeler, J. A., \& Zurek, W. H. (2014). Quantum theory and measurement. Princeton University Press.

[12]Williams, D. B., \& Carter, C. B. (1996). The transmission electron microscope (pp. 317). Springer US.

[13]Zhang, J. Z. (2009). Optical Properties and Spectroscopy of Nanomaterials. USA.

\section{How to cite this article:}

Karami A and Badiee D. Designing a curriculum about electron microscope based on disciplinary method for graduate students. J. Fundam. Appl. Sci., 2016, 8(3S), 501-508. 